\title{
Praxiserfahrung mit dem IQM-Projekt aus Sicht eines freigemeinnützigen Trägers
}

Die Arbeit mit Qualitätsindikatoren und die Umsetzung des IQM-Projektes erfolgten bereits 2008 im Klinikum Straubing.

Die praktischen Erfahrungen unseres Trägers mit dem IQM-Projekt und den verwendeten Indikatoren-Sets berühren folgende 5 Aspekte:

- Praktische Unterstützung der Geschäftsführung bei der strategischen Planung. In der Vergangenheit stützte sich die strategische Planung der Krankenhäuser vorwiegend bis ausschließlich auf Finanz- und Leistungskennzahlen. Besonders in einem sich sehr rasch verändernden Umfeld, wie es derzeit in der Gesundheitsbranche der Fall ist, vermögen solche vergangenheitsbezogenen Daten nur sehr bedingt Aussagen zur näheren Zukunft zu geben. Daher müssen für die strategische Planung der Krankenhäuser zunehmend zukunftsgewandte Kennzahlen verwendet werden und in erster Linie Daten zu Patientenoutcome, da sich hier „das Kerngeschäft des Krankenhauses" abbildet.

- Sicherstellung einer zielgerichteten Ressourcen-Allokation.

In Zeiten gedeckelter Ressourcen, bei gleichzeitig deutlich steigenden Kosten stellt sich in immer virulenterer Form die Frage, wie die begrenzten Ressourcen genutzt und verteilt werden. Gerade für konfessionelle Häuser geht es um mehr als nur eine Methode der Ressourcen-Allokation, sondern um die Frage der Allokationsethik, nach der gearbeitet wird. Qualitätsindikatoren, wie sie auch im $\mathrm{IQ}^{\mathrm{M}}$-Projekt vertreten sind, können daher genutzt werden, um ein intelligentes und transparentes System der Ressourcen-Allokation einzurichten.

- Eckpfeiler des Riskmanagements des Krankenhauses.

Riskmanagement im Krankenhaus kann sehr unterschiedlich definiert und umgesetzt werden. Hauptforderung jedoch an ein Riskmanagement im Krankenhaus sollte sein, dass es „das Kerngeschäft“ betrifft, eine Alarmfunktion sicherstellt und Ressourcen-schonend umgesetzt werden kann. Die Erhebung von Qualitätsindikatoren mit Routinedaten, wie sie im IQM-Projekt verwirklicht ist, erfüllt diese Kriterien und hat dazu geführt, dass diese Daten zu einem wesentlichen Element des Riskmanagements des Trägers geworden sind.

- Qualitätssteuerung des Krankenhauses. Qualitätsindikatoren und Qualitätsberichte können nur so gut sein, wie sie genutzt werden, um kontinuierliche Verbesserungen im Krankenhaus auszulösen. Die quartalsmäßige
Analyse der Qualitätsindikatoren ist bei den oft geringen Fallzahlen mit Vorsicht vorzunehmen, erlaubt aber dank Einzelfallanalysen rasche unterjährige Korrekturen. Voraussetzung für die zügige Umsetzung in die Praxis ist neben einem bedingungslosen Commitment der Geschäftsführung, eine breite Kommunikation zu den internen Leistungsträgern (Leitungskonferenz) wie auch zu allen Mitarbeitern des Krankenhauses (offene Klinikumskonferenz).

- Einbindung in die Kommunikationsstrategie des Krankenhauses.

Patienten, Angehörige, Krankenkassen sowie Politik und Gesellschaft haben ein sehr großes Bedürfnis nach Daten und Fakten zur Ergebnisqualität von Leistungserbringern im Gesundheitssystem. Die Komplexität der klinischen Wirklichkeit und die schiere Anzahl an Indikatoren lässt es opportun erscheinen, neben einer allgemeinen Kommunikation der Jahreswerte für alle Indikatoren gezielt auch die Ergebnisse für Zielgruppen aufzuarbeiten (z.B. Selbsthilfegruppen). Erfahrungen zur Kommunikation mit solchen Focus-Gruppen bestehen im Bereich Schlaganfall, Endoprothetik oder im Rahmen eines Darmzentrums.

Autorenerklärung: Der Autor erklärt, dass keine relevanten finanziellen Verbindungen in Bezug auf dieses Manuskript bestehen.
C. Scheu

Qualitätsmanagement

Schlüsselwörter

OIQM

Qualitätsindikatoren

Gemeinnützigkeit

Keywords

O IQM

quality indicators

non-profit hospital

(1)

Dol 10.1055/s-0029-1242669

Dtsch Med Wochenschr 2009; 134: S313 - (c) Georg Thieme Verlag KG Stuttgart · New York . ISSN 0012-0472

Korrespondenz Dr. Christoph Scheu, MBA Klinikum St. Elisabeth Straubing St. Elisabeth-Straße 23 94315 Straubing Tel. 09421/710-1020 eMail christoph.scheu@ klinikum-straubing.de 